\title{
Laser-Assisted Cryosurgery in ex vivo Mice Hepatic Tissue: Viability Assays Using Green Fluorescent Protein
}

\author{
L. Martínez-Suástegui, ${ }^{1,4}$ B. Duperray, ${ }^{2}$ F. Godinez, ${ }^{1}$ G. Guillén, ${ }^{3}$ A. Slade, ${ }^{1}$ and G. Aguilar ${ }^{1}$ \\ ${ }^{1}$ Department of Mechanical Engineering, University of California, Riverside, CA 92521, USA; ${ }^{2}$ Ecole Nationale Superieure \\ d'Ingenieur de Caen, Caen 14000, France; ${ }^{3}$ Departamento de Biología Molecular de Plantas, Instituto de Biotecnología, UNAM, \\ Cuernavaca 62210, Mexico; and ${ }^{4}$ Sección de Estudios de Posgrado e Investigación-IPN, México, DF 02250, Mexico
}

(Received 7 May 2010; accepted 9 October 2010; published online 21 October 2010)

Associate Editor Kyriacos A. Athanasiou oversaw the review of this article.

\begin{abstract}
An experimental investigation is carried out to develop a novel approach to cryosurgery, where laser heating counteracts tissue freezing to better confine damage to the targeted cancerous tissue within a lethal low-temperature isothermal boundary - an approach we refer to as laserassisted cryosurgery (LAC). The advantage of this procedure relative to conventional cryosurgery assisted with urethral warmers or cryoheaters is that laser heating provides volumetric rather than superficial heating, which leads to deeper penetration, more homogeneous tissue protection and better demarcation of the destructive freezing effect to a welldefined targeted volume. Tissue viability assays are performed using green fluorescence protein (GFP) as a viability marker and correlated with temperature history after performing LAC procedures on ex vivo mice hepatic tissue. The limit for cell denaturation at the irradiated surface predicted by GFP analysis is further confirmed using reverse transcription polymerase chain reaction (RT-PCR). In addition, the correlation between GFP fluorescence and cell viability and loss of GFP fluorescence in non-viable cells has been tested and validated by histological analysis using a standard cell viability measuring method (hematoxylin and eosin staining). Analysis of our experimental measurements show that reproducible thermal gradients (of $236{ }^{\circ} \mathrm{C} / \mathrm{cm}$ ) and predictable tissue necrosis can be reliably produced by LAC without exceeding temperature thresholds for cell denaturation (of $T_{\text {surf }} \approx 48{ }^{\circ} \mathrm{C}$ ) beyond preset tissue boundaries (with resolution of $0.1^{\circ} \mathrm{C} / \mathrm{mm}$ ). The results have shown the feasibility of controlling temperatures at specified tissue locations to prevent hyperthermal or freezing damage.
\end{abstract}

Keywords-Cryoablation, Prostate cancer, RT-PCR.

Address correspondence to L. Martínez-Suástegui, Sección de Estudios de Posgrado e Investigación-IPN, México, DF 02250, Mexico. Electronic mail: martinezlorenzo@gmail.com

\section{NOMENCLATURE}

$\begin{array}{ll}d & \text { Distance }(\mathrm{mm}) \\ \text { SD } & \text { Standard deviation } \\ t & \text { Time }(\mathrm{s}) \\ T & \text { Temperature }\left({ }^{\circ} \mathrm{C}\right) \\ \text { TC } & \text { Thermocouple }\end{array}$

Greek Letters

$\delta \quad$ Thickness of protection layer (mm)

$\mu_{\mathrm{a}} \quad$ Absorption coefficient $\left(\mathrm{mm}^{-1}\right)$

\section{Subscripts}

Amb Ambient

Cryo Cryoprobe

Surf Surface

Th Threshold

\section{INTRODUCTION}

Cryosurgery is the infliction of a lethal freezing injury achieved by the contact of one or more metallic probes at low subzero temperatures with a target tumor tissue. Prostate cryosurgery was the first minimally invasive cryosurgical procedure to pass from the experimental stage and become a routine surgical treatment. ${ }^{25}$ While the main objective during a cryosurgical procedure is to ensure tissue destruction within the cryolesion, the greatest challenge is on how to spare the surrounding healthy tissues from cryoablation. In order to overcome this difficulty and with the aid of recent technological developments, the total number of cryoprobes used during a cryosurgical procedure has been increased because of a considerable 
reduction in cryoprobe size and an increase in the ability to control the freezing rate. ${ }^{6,27,30}$ Recent improvements in imaging devices that help to monitor the freezing process, such as ultrasound or MRI, have further enhanced the popularity of cryosurgery. ${ }^{16,18,30,36,37}$ Also, numerical optimization techniques have been developed ${ }^{1,2,19,36,37}$ so that the optimal number and placement of cryoprobes minimizes collateral damage.

During prostate cryosurgery, urethral warmers (double-pipe water heat exchangers embodied in a standard catheter) are used for routine cryosurgery control $^{8,28}$ to protect the urethral wall from damage. A recent investigation suggests that cryoheaters (temperature-controlled electrical heaters) can be more effective in protecting the urethral surface and better shaping the frozen region to maximize destruction of the unhealthy tissue. ${ }^{29}$ Although this may greatly improve the procedure effectiveness, the success of cryosurgery is still very dependent on the experience and good judgment of the surgeon, who monitors the growth of the ice ball and determines its maximum size. ${ }^{20}$ In this regard, if temperatures were maintained above the damage threshold within healthy tissue at all times, independent of the cooling application time, great progress would be achieved.

Recently, a new means of cryosurgery control was suggested, ${ }^{31,32}$ where laser heating counteracts tissue freezing to better confine damage to the targeted cancerous tissue within a lethal low-temperature isothermal boundary - an approach referred to as laserassisted cryosurgery (LAC). The thermal confinement achieved by LAC was compared against that achieved by warmers or cryoheaters, demonstrating that LAC was more efficient in terms of protecting a much thicker layer of tissue. In LAC, the frozen region can be shaped to selectively destroy malignant tissue. This is achieved by strategically inserting minimally invasive optical fibers into predetermined sites to supply the volumetric heating needed to prevent unwanted tissue freezing. This approach is very practical for worst case scenario for tissue damage, which occurs when the steady-state temperature is reached in the region of interest, which is when the freezing front gets closer to the heating boundary. The advantage of LAC compared with traditional methods is that it provides protection to certain regions of tissue from being frozen without limiting so severely the application of the cryoprobe. In addition, if only a portion of the prostate needs to be destroyed, cryoablation of portions of healthy prostate tissue can be avoided by effectively localizing optical fibers inside the prostate. This approach cannot be employed using cryoheaters or warmers. The major impact of a successful LAC procedure is that there would be no need to stop the freezing process prior to achieving the target lethal temperature. In addition, a more uniform and lower temperature inside the tumor could be achieved by freezing to temperatures below the lethal isotherm ${ }^{4,12}$ and effectively confining the frozen tissue within preset boundaries.

The correlation between loss of GFP fluorescence and loss of cell viability is well established and has been previously tested and validated. ${ }^{17}$ GFP was used to monitor metabolic activity and the authors point out that this detection method should be used as a standard assay. ${ }^{38}$ The GFP-based assay was compared against commercial viability DNA-binding dyes (SYTO9-PI), and results showed that the GFP-based assay improves reliability for the assessment of cell viability with no marked difference in sensitivity. ${ }^{18}$ Also, GFP was used as a reporter for cell viability and the authors point out that it offers greater simplicity, easier monitoring, enhanced biosafety, and lower cost than the luciferase reporter assay. ${ }^{9}$ The results obtained with the GFP viability assay method were confirmed by comparing them against Propidium Iodide and Annexin V staining. ${ }^{35}$ The authors reported that GFP can be used as an excellent marker for detection of necrosis by measuring the decrease of GFP fluorescence intensity. In addition, the validity of GFP viability assays for cryobiological applications has been established by comparing the results against common viability indicators (trypan blue and ethidium bromide) with excellent correlation coefficients of 0.97 and 0.95 , respectively. ${ }^{11}$ As GFP accumulates in the cytoplasm of the normally metabolizing transfected cells, damage to the plasma membrane due to heating or freezing insults results in leakage of cell contents, including GFP. ${ }^{3}$ Denaturation of the GFP results in a loss of fluorescence, primarily because the chromophore is no longer encapsulated and protected., 26 Since viable cells express GFP continuously, loss of GFP fluorescence in necrotic cells is used to correlate the recorded temperature histories with the outcome of viability after a LAC procedure.

The aims of the present investigation are to (a) assess the feasibility of LAC to limit cryoinjury to a predefined region and (b) correlate tissue viability with local tissue temperature histories on ex vivo mice hepatic tissue after exposed to LAC procedures. For this purpose, we use mice transfected with green fluorescence protein (GFP). As GFP fluorescence in nonviable cells is lost when cell membranes are damaged either by freezing or heating insults, GFP is used as a marker for tissue viability by relying on the expression and retention of GFP. The maximum temperature at which thermal necrosis occurs at the irradiated surface during LAC is further confirmed using reverse transcription polymerase chain reaction assays. 
The ultimate applicability of this research is to destroy with precision any tumor regardless of its size and shape by avoiding postsurgical complications and malignancy recurrence.

\section{MATERIALS AND METHODS}

\section{Sample Preparation}

LAC was performed ex vivo on fresh mouse liver samples. Cryothermia was induced to the liver of transgenic mice expressing GFP in all of their cells using a $7.7-\mathrm{mm}$-diameter cylindrical cryoprobe cooled by internally circulating liquid nitrogen. The temperature of the cryoprobe tip $\left(T_{\text {cryo }}=-188^{\circ} \mathrm{C}\right)$ was determined by placing a thermocouple at the tip's surface. For freezing, the cryoprobe was placed carefully on the surface of one side of the tissue sample after being precooled. A Nd:Yag Laser system (CoolTouch, NS130C) with a wavelength of $1320 \mathrm{~nm}$ and a maximum power of $10 \mathrm{~W}$ was used to irradiate and induce volumetric heating to the tissue from one end. For these experiments, the laser light was attenuated with optical density filters to the required energy $(43 \mathrm{~mJ}$, pulse duration $300 \mu \mathrm{s}, 20 \mathrm{~Hz}$, spot size $2.6 \mathrm{~mm}$ ). The absorption coefficient in mouse hepatic tissue for a wavelength of $1064 \mathrm{~nm}$ is of $\mu_{\mathrm{a}}=0.13$ $\left(\mathrm{mm}^{-1}\right) \cdot{ }^{24}$ Although an exact assessment of the optical properties of mouse hepatic tissue was not performed for a wavelength of $1320 \mathrm{~nm}$, since in the near-infrared the absorption decreases with shorter wavelengths due to tissue water content, the thermocouples were not considered as light absorbers because they were inserted at a distance from the irradiated surface that's larger than the penetration depth of light in mouse liver tissue at a wavelength of $1320 \mathrm{~nm}$.

The resultant temperature histories produced within the tissue specimens during a LAC procedure were determined experimentally with an array of four separate 33-gage needle copper-constantan thermocouple probes (Omega Engineering, HYP0 mini-hypodermic probes) connected to a data acquisition system. The thermocouple probes were held in position by an acrylic lid, and the vertical positions of the thermocouples were adjusted with depth stops so that all tips were brought to the same plane orthogonally to the lid at the depth of the laser lightguide axis. The in situ temperature measurements were acquired at $2.5 \mathrm{~Hz}$, displayed in real-time and logged into a PC for further analysis. Figure 1 shows a schematic diagram of the experimental setup used to measure the temperature response of tissues at selected points during a LAC procedure.

\section{LAC Procedure}

All procedures employed in this study were approved by the Institutional Animal Use and Care Committee of the University of California, Riverside: Protocols $A-20080013$ and $A-20080019$. After the animals were euthanized by $\mathrm{CO}_{2}$ gas asphyxiation, the liver was excised and carefully cut with a custom cutting tool to a predetermined rectangular block of $10 \times 6 \times 2.6 \mathrm{~mm}$.

Hepatic tissue was selected because it is one of the largest organs of the mouse. A tissue block size of $1 \mathrm{~cm}$ was chosen because it allowed measurements of the temperature history at four different locations inside the tissue sample to be taken. Rectangular tissue

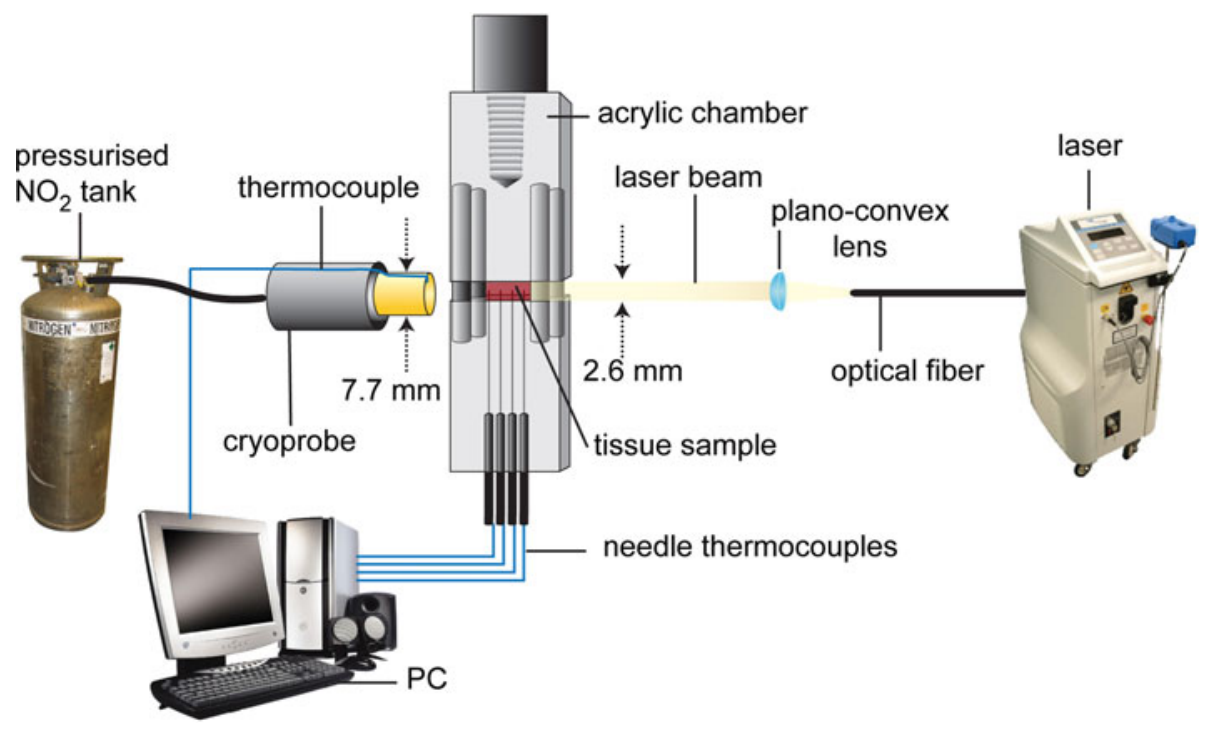

FIGURE 1. Schematic of the experimental setup. 
(a)

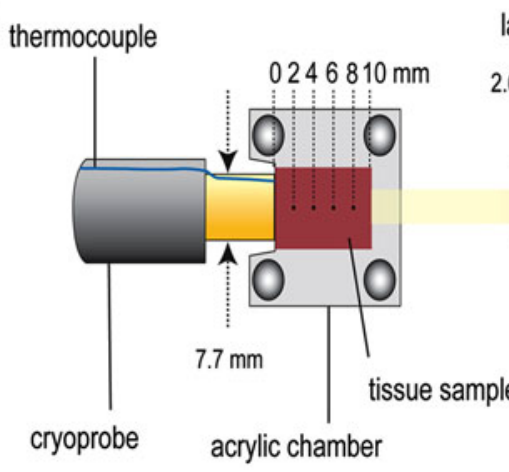

laser beam

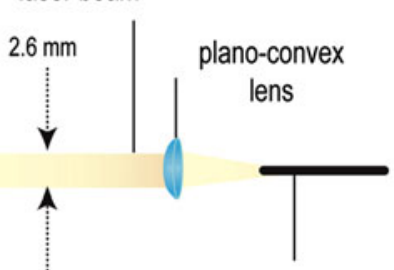

optical fiber

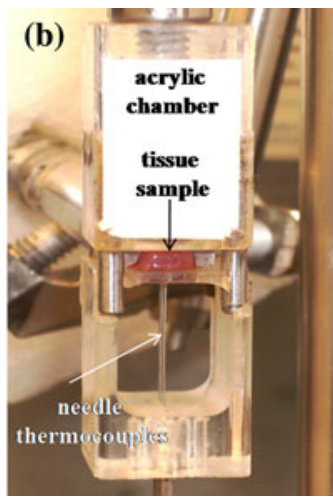

FIGURE 2. (a) Disassembled acrylic chamber (upper view) with the miniature needle thermocouples at even and known distances. (b) After the tissue sample is placed and the thermocouples inserted, the chamber is closed.

samples were used for all the experiments in order to avoid variations in their dimensions that could lead to significant variations of the position of $T_{\text {th }}$ from one experiment to the other. ${ }^{31,32}$ The tissue sample was carefully placed and fitted with the miniature needle thermocouples in the disassembled transparent acrylic chamber shown in Fig. 1 and detailed in Fig. 2. The needle thermocouples $\mathrm{TC}_{1}, \mathrm{TC}_{2}, \mathrm{TC}_{3}$, and $\mathrm{TC}_{4}$ were mounted equidistant from the cryoprobe tip at: $d=2$, 4,6 , and $8 \mathrm{~mm}$, respectively. Afterward, the chamber was closed and the tissue sample was slightly compressed so that there was no variance in its dimensions from one experiment to another. The cryolesion was created using a single freeze/thaw cycle, and throughout the experiment, ice ball progression was monitored with a digital camera. The temperature data from the LAC procedure was compiled to evaluate the freezing front propagation and to correlate the thermal history with the resulting cryoinjury, seeking to define the so called "lethal temperature."

\section{Reverse Transcriptase Polymerase Chain Reaction Analysis}

To quantify the amount of hyperthermic damage induced to the cells at the irradiated surface for different laser settings, reverse transcriptase polymerase chain reaction (RT-PCR) analysis was performed. To test the integrity of the RNA of LAC-treated samples and untreated controls, RNA was isolated from $100-\mu \mathrm{m}$ thick slices taken from the laser-irradiated surface after $2 \mathrm{~h}$ of being irradiated and RNA was extracted using the QIAGEN RNeasy ${ }^{\circledR}$ mini kit (QIAGEN) according to the instructions of the manufacturer. RNA yield and purity was analyzed using a NanoDrop 1000 spectrophotometer. RT-PCR expression of $\beta$-actin was determined in tissue samples after LAC procedures and appropriate controls using conventional RT-PCR. Briefly, 50 ng total RNA was reverse transcribed using oligo $(\mathrm{dT})^{15}$ primer, $1 \mathrm{U}$ of RNasin ribonuclease inhibitor and $1 \mathrm{U}$ of $\mathrm{AMV}$ reverse transcriptase system (Promega ${ }^{\mathrm{TM}}$ ) for $10 \mathrm{~min}$ at $70{ }^{\circ} \mathrm{C}, 5 \mathrm{~min}$ at $4{ }^{\circ} \mathrm{C}, 60 \mathrm{~min}$ at $42{ }^{\circ} \mathrm{C}$, and $5 \mathrm{~min}$ at $95^{\circ} \mathrm{C}$. A $154 \mathrm{pb}$ fragment of $\beta$-actin was amplified with Taq DNA polymerase (Biolabs) using $100 \mathrm{ng}$ of each cDNA and $300 \mu \mathrm{M}$ primers (forward primer: 5'-CCTCTATGCCAACACAGT-3'; reverse primer: 5'-AGCCACCAATCCACACAG-3'). PCR conditions were based on the recommendations of the manufacturer. An initial cycle at $95{ }^{\circ} \mathrm{C}$ for $1 \mathrm{~min}$, followed by 35 cycles of $30 \mathrm{~s}$ at $95{ }^{\circ} \mathrm{C}, 30 \mathrm{~s}$ at $55^{\circ} \mathrm{C}$, and $120 \mathrm{~s}$ at $60{ }^{\circ} \mathrm{C}$. The amplicon was resolved with electrophoresis on agarose gel $(2 \%)$ and stained with ethidium bromide. A target temperature of $3{ }^{\circ} \mathrm{C}$ for $\mathrm{TC}_{4}$ was found to be the value that maximizes the thickness of the protection layer without surpassing the lethal threshold for cell viability due to hyperthermia.

\section{Time Sequence for a LAC Procedure}

The complete LAC procedure lasts a total of $700 \mathrm{~s}$ and consists of a single freeze/thaw cycle. The freezing process involves a single 300-s freeze period (consisting of an initial 200-s transient and a 100-s steady-state) followed by a 400 -s thawing period. Regulated laser power was supplied during the transient freezing and thawing periods, while constant laser power was supplied during steady-state heat transfer. After thawing, the samples were stored in Dulbecco's Phosphate Buffered Saline (DPBS) and analyzed for viability using GFP as a viability marker.

\section{Power Regulation/Modulation and Establishment of Steady-State}

During LAC, the power needed to achieve the desired protection depends on the thermal/optical 
properties of the tissue and the separation between the cryoprobe and the laser-irradiated surface. Since the time scale for energy transport by conduction in tissue is different to that for laser energy absorption, the cryoprobe and the laser must be turned on at different times and the laser energy must be supplied gradually during the procedure. For simplicity, during a LAC procedure the laser power delivered remains fixed to a preset value and, to avoid undesirable heating damage, the laser beam is blocked using a mechanical device that works as a shutter that switches the laser on or off. The appropriate timing for laser irradiation/suppression was designed to maintain the maximum tissue temperature below that known to produce thermal necrosis $\left(43-48{ }^{\circ} \mathrm{C}\right) \cdot{ }^{21,22,33,34}$ While the maximum temperature was expected to be reached at or near the irradiated surface, it was impractical to use a thermocouple placed at that surface for the laser control feedback because the bead absorbs the laser light directly and, thus, disrupts the temperature field at that location. Instead, this temperature feedback was obtained by using the reading of the thermocouple located farthest away from the cryoprobe tip $\left(\mathrm{TC}_{4}=8 \mathrm{~mm}\right)$. Preliminary tests showed that a target temperature of $\mathrm{TC}_{4}=3{ }^{\circ} \mathrm{C}$ was required to maximize the thickness of the protected tissue and to avoid loss of fluorescence due to undesired thermal damage at the irradiated surface. The temperature of the irradiated surface remained within a viable value (presumably less than $\sim 48{ }^{\circ} \mathrm{C}$ ), as was confirmed by RT-PCR analysis performed at the irradiated surface (see Section Reverse Transcriptase Polymerase Chain Reaction (RT-PCR) Analysis).

The laser power requirements needed to achieve the desired protection in the implementation of LAC had already been addressed. ${ }^{32}$ Briefly, the cryoprocedure starts by turning the cryoprobe on to initiate the freezing process while the laser is off. During the transient process, as the freezing front advances away from the cryoprobe and the temperature at the opposite end starts to drop, regulated laser irradiation is gradually applied to avoid excessive heating. As a consequence of the progressive increase in laser irradiation heating, the freezing front is stopped at some distance from the irradiated surface and a steady-state temperature distribution is achieved and held by supplying constant laser irradiation while maintaining a target temperature of $3 \pm 1{ }^{\circ} \mathrm{C}$ in $\mathrm{TC}_{4}$. For the last stage of the cryoprocedure, the cryoprobe is turned off and the regulated laser irradiation supplied is gradually decreased so that the target temperature in $\mathrm{TC}_{4}$ is always maintained. Afterward, the laser is turned off and the sample is thawed at room temperature until there are no visible signs of frozen tissue. Figure 3 shows the normalized laser

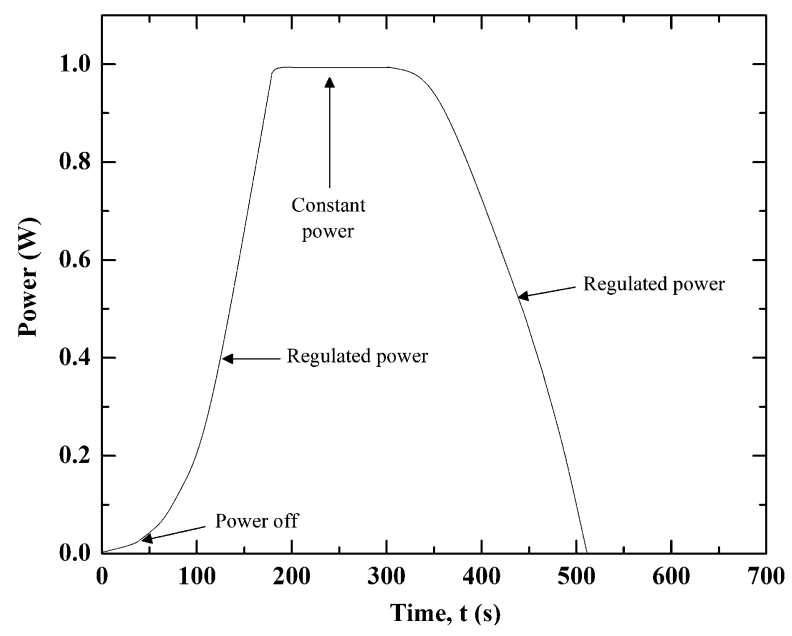

FIGURE 3. Normalized laser power as a function of time required to avoid excessive heating at the irradiated surface during a complete LAC procedure with a total duration of 700 s starting when the cryoprobe is turned on to initiate the freezing process. For clarity, the variations in the laser power supplied by blocking the laser beam with a shutter are not shown.

power as a function of time required during a complete LAC procedure in order to avoid undesirable heating at the irradiated surface. Note that although laser power regulation is performed by blocking the laser beam with a shutter, this method is sufficient to control the temperature of the tissue sample without producing any significant temperature fluctuations in the overlying volume. Nevertheless, future work in the implementation of LAC must be aimed toward the development of an accurate laser power algorithm that includes a control approach with optimization.

\section{GFP Viability Assays}

Expression of GFP, originally isolated from the bioluminescent jellyfish Aequorea victoria, represents a unique method for fluorescent labeling of viable cells. ${ }^{5}$ An advantage of using GFP fluorescence is that the tissue can be assayed without altering the system in any way, other than illuminating with light of the proper excitation wavelength. GFP fluorescent assays do not require staining or fixation of the tissue to indicate viability, but rather rely on the expression of GFP, where aside from the addition of buffer substrate, no other processing of the sample is required.

In this work, GFP viability assays are performed following a LAC procedure by using transgenic mice expressing GFP in all tissues obtained from The Jackson Laboratories (Bar Harbor, MD) to accurately quantify the limits of tissue viability. 


\section{RESULTS}

\section{Temperature Recordings}

All of the LAC procedures presented were performed using the time sequence previously described. Figures $4 \mathrm{a}-4 \mathrm{e}$ show the elapsed time required for the freezing front to travel from the cryoprobe tip to the sites where the thermocouples were located. Figure $4 f$ shows how the freezing front is stalled during steadystate heat transfer (from $200 \leq t \leq 300 \mathrm{~s}$ ) at a distance of $d \approx 8.2 \mathrm{~mm}$ away from the cryoprobe.
Figure 5 shows the recorded temperature history for a LAC procedure. The tissue temperatures measured at various longitudinal positions illustrate the temperature gradient achieved. The lowest temperature inside the tissue is achieved at a distance of $d=0 \mathrm{~mm}$ at the cryoprobe tip, while the higher temperature shown for reference is the ambient temperature $T_{\mathrm{amb}}=25^{\circ} \mathrm{C}$.

Figure 6 shows the temperature profile of the tissue sample during steady-state heat transfer. The temperatures shown are expressed as mean \pm SD for $200 \leq$ $t \leq 300 \mathrm{~s}$. The mean and SD were calculated with the (a)

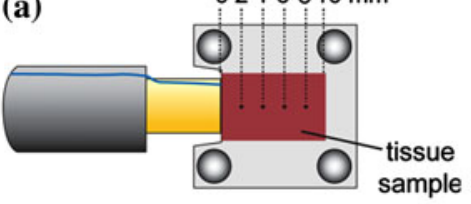

$\mathrm{t}=0 \mathbf{s e c}$

(d)

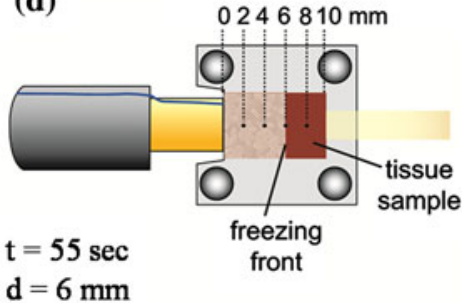

(b)

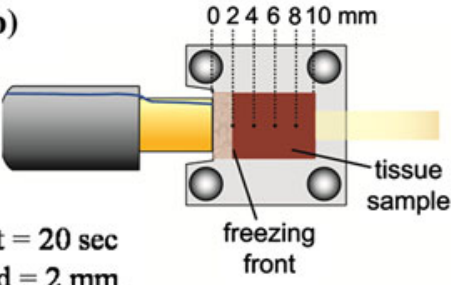

(e)

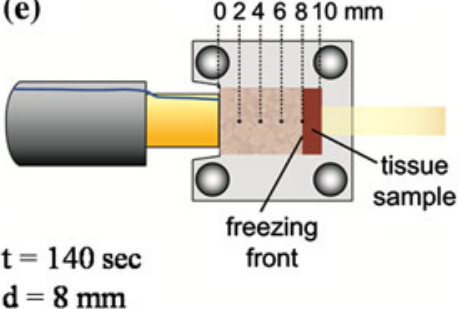

(c)

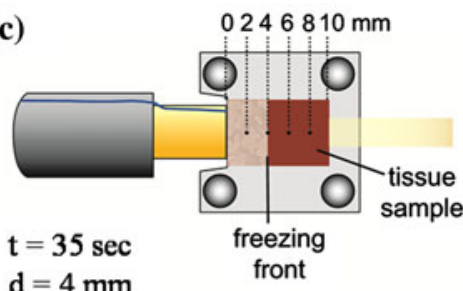

(f)

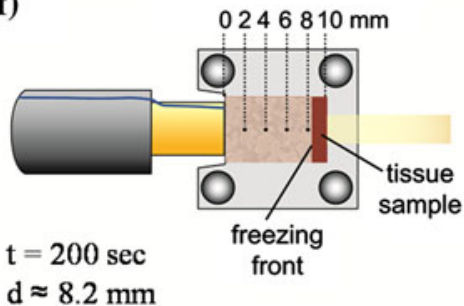

FIGURE 4. (a)-(e) The images illustrate the freezing front advancement during a LAC procedure from the cryoprobe tip at: $d=0$, $2,4,6$, and $8 \mathrm{~mm}$, respectively. (f) From $200 \leq t \leq 300 \mathrm{~s}$ the freezing front is stalled at $d \approx 8.2 \mathrm{~mm}$ away from the cryoprobe during steady-state heat transfer.

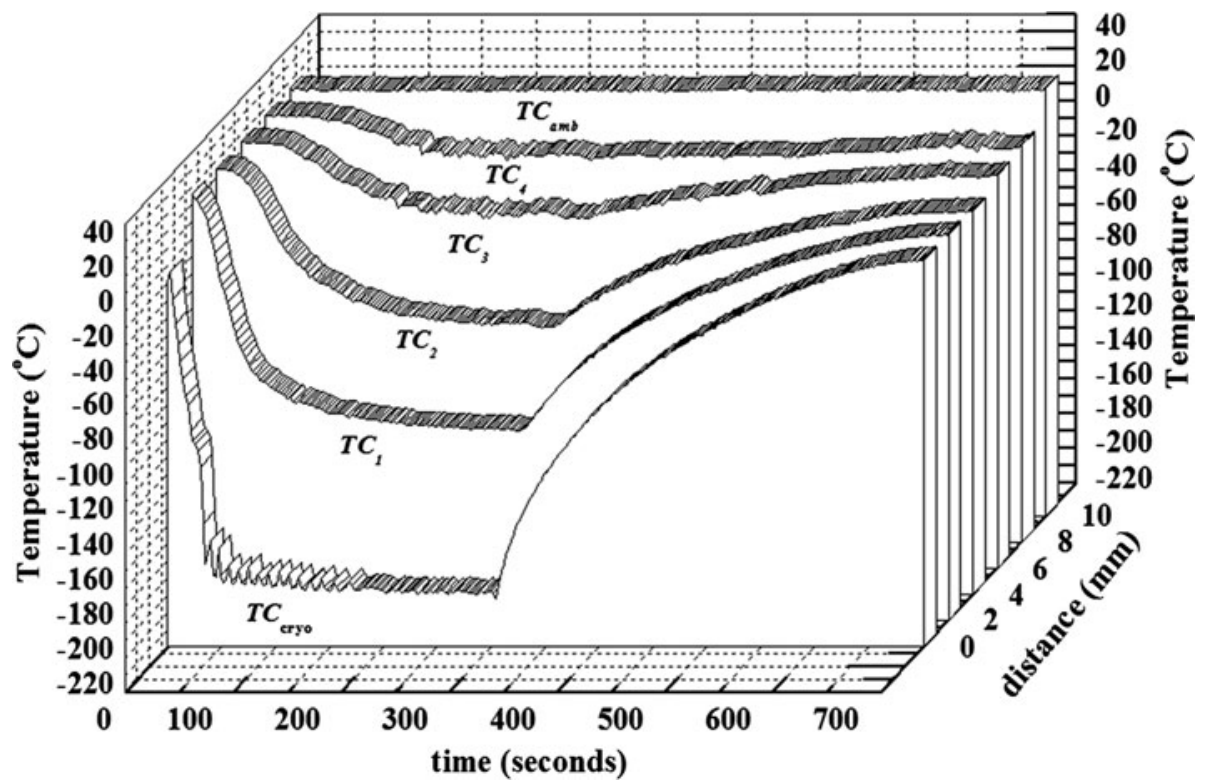

FIGURE 5. Tissue temperature measured by the five thermocouples at various longitudinal positions as a function of time during a LAC procedure. The closer the thermocouples to the cryoprobe are, the steeper is the fall of the tissue temperature upon freezing. 


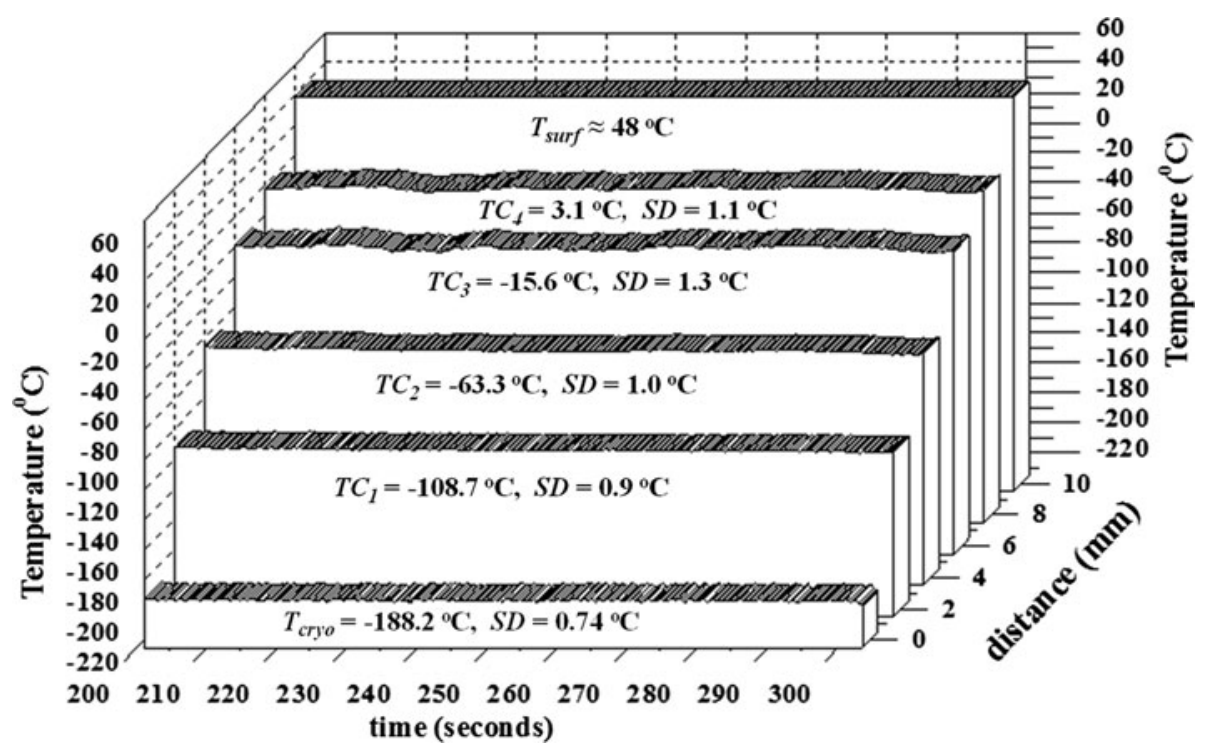

FIGURE 6. Temperature history during steady-state heat transfer. The temperatures displayed are expressed as mean \pm SD for $200 \leq t \leq 300 \mathrm{~s}$.

readings of the thermocouples for each time instant and the sample size was of 250 for each thermocouple. Note that the temperature gradients are very steep, e.g., having a temperature of $T_{\text {cryo }}=-188{ }^{\circ} \mathrm{C}$ at the cryoprobe tip while a maximum surface temperature of $T_{\text {surf }}$ at a distance of $10 \mathrm{~mm}$ away from the cryoprobe remained within a viable value throughout the whole LAC procedure. ${ }^{21,22,33,34}$ Freezing with a shorter interval was not performed since the temperature profiles during steady-state would be the same regardless of the duration of the freezing interval. Correlation between the temperature histories and the outcome of viability assays showed that the threshold of lethal injury after a LAC procedure $\left(T_{\text {th }} \approx 4{ }^{\circ} \mathrm{C}\right)$ was located at a distance of $d \approx 8.2 \mathrm{~mm}$ away from the cryoprobe. Note that this value is higher than the lethal threshold for conventional cryosurgical procedures (where the lethal threshold occurs over a range of temperatures from 0 to $-50{ }^{\circ} \mathrm{C}$ ).

An analysis of the reproducibility of the resultant temperature distribution was carried out from repeated experiments on ex vivo mice hepatic tissue. The resultant temperature profiles in this series of experiments showed excellent reproducibility, with an error of $<4 \%$ in the averaged mean target temperatures and a SD of $<1.4{ }^{\circ} \mathrm{C}$. The feasibility of LAC to limit cryoinjury to a predefined region has been assessed after correlating tissue viability with local tissue temperature histories on ex vivo mice hepatic tissue after exposed to LAC procedures. Results show that reproducible thermal gradients (of $236^{\circ} \mathrm{C} / \mathrm{cm}$ ) and predictable tissue necrosis can be reliably produced by LAC without exceeding temperature thresholds for cell denaturation
TABLE 1. Results of mean RNA yields between LAC-treated samples and untreated controls expressed as mass of RNA in $\mathrm{ng} / \mu \mathrm{L}$.

\begin{tabular}{lccc}
\hline Sample & $\begin{array}{c}\text { Normalized } \\
\text { mass of } \\
\text { RNA in } \mathrm{ng} / \mu \mathrm{L}\end{array}$ & $\begin{array}{c}\text { Standard } \\
\text { error of the } \\
\text { mean (SEM) }\end{array}$ & $\begin{array}{c}\text { GFP fluorescence } \\
\text { at the irradiated } \\
\text { surface }\end{array}$ \\
\hline Control & 1 & 0.000 & Yes \\
$\mathrm{TC}_{4}=3{ }^{\circ} \mathrm{C}$ & 0.963 & 0.013 & Yes \\
$\mathrm{TC}_{4}=6{ }^{\circ} \mathrm{C}$ & 0.455 & 0.021 & No \\
$\mathrm{TC}_{4}=35^{\circ} \mathrm{C}$ & 0.061 & 0.008 & No \\
Inverse control & 0.066 & 0.001 & No \\
\hline
\end{tabular}

The samples are $100-\mu \mathrm{m}$ thick slices taken from the laserirradiated surface and inverse controls were taken from the surface where the cryoprobe was placed. Data represent averages of three replicates. LAC-treated samples show the target temperature used for $\mathrm{TC}_{4}$.

(of $T_{\text {surf }} \approx 48^{\circ} \mathrm{C}$ ) beyond preset tissue boundaries (with resolution of $0.1{ }^{\circ} \mathrm{C} / \mathrm{mm}$ ).

Table 1 shows a comparison between the results of RNA yields for LAC-treated samples and untreated controls for different values of the target temperature measured at $\mathrm{TC}_{4}$. The inverse controls were taken from the surface where the cryoprobe was placed. Three replicates of this assay were performed and the values displayed are normalized values of mean mass of RNA in $n g / \mu \mathrm{L}$, the standard error of the mean (SEM), and the resultant GFP fluorescence. In addition, $150 \mathrm{pb}$ are amplified from the $\beta$-actin gene. The RT-PCR results show that $\beta$-actin expression is detectable and virtually identical between irradiated and non-irradiated tissues. 


\section{Viability Assays}

After thawing, the samples were stored in DPBS and assessed with a Leica MZIII Stereo Dissection Microscope (Diagnostic Instruments, Inc.) coupled to a digital color/grayscale camera (Pursuit $4 \mathrm{Mp}$ Slider, Model 16.4) by detecting fluorescence under UV illumination using Spot Advance software (version 4.6). The recorded images were then analyzed to determine with precision the thickness of the protected region due to laser irradiation, the extent of necrotic tissue due to cryoablation, and the temperatures below which tissue was cryoablated.

In order to avoid false positive viability assessment due to a narrow time window after thermal insult, the appropriate time point needed for lethally affected cells to lose fluorescence was estimated for both hyperthermia and cryoablation. Surprisingly, it was found that they differ considerably. For the case of temperatures above the lethal threshold for cell denaturation due to hyperthermia, GFP was immediately degraded and fluorescence was immediately lost. On the other hand, it was found that the damage induced by freezing was not evident immediately after a LAC procedure. Therefore, the sample had to be stored in DPBS at room temperature for $5 \mathrm{~h}$ before being imaged so that the transient changes in permeability suggesting lethal damage lead to loss of GFP fluorescence. This is a very important issue in cryobiology applications. It was found that this time window after the thermal insult was needed after cryoablation. In order to make sure that fluorescent cells after the time window are positively alive, the results obtained with the GFP viability assay method were confirmed by comparing them against histological analysis using a standard cell viability method (hematoxylin and eosin staining). Tissue samples were divided into two main treatment groups: untreated controls and tissue samples that underwent a LAC procedure without laser heating. Both groups were incubated using the same protocol described above. After the incubation period, tissue samples from both treatment groups were analyzed for viability using GFP fluorescence as a viability marker. After verifying that the control was fluorescing brightly and that the treated tissue samples had lost fluorescence due to damage induced by cryoinjury, formalin fixation was performed. Liver sections $(10 \mu \mathrm{m})$ were prepared using a microtome and placed on glass slides. Standard hematoxylin and eosin (H\&E) stains were employed and histopathologic examination was determined by light microscopy. Representative sections from $H \& E$ staining in mouse hepatic tissue are shown in Fig. 7. The histologic appearance of the liver parenchyma in the control group appeared normal and showed regular hepatocellular architecture with intact sinusoidal lining. The structural details are shown in Fig. 7a. The tissue samples that underwent a LAC procedure without laser heating revealed homogeneous severe tissue damage and necrosis at all sites. Figure $7 \mathrm{~b}$ illustrates how exposure to temperatures below the lethal isotherm caused a destructive effect in the liver with clear histopathological alterations such as sinusoidal congestion, hepatocyte congestion, and loss of cellular morphology. The findings of the present histological investigations corroborated a direct correlation between fluorescent tissue and viable tissue after the time window. In addition, massive hepatic necrosis indicating irreversible cell death was apparent in all of the cryotreated tissue samples that had lost fluorescence after the incubation period.

Figure 8 shows the outcome of viability following LAC. Figures $8 \mathrm{a}$ and $8 \mathrm{~b}$ are two different samples assayed after identical LAC procedures. On the left side of the sample, a scale displays the thickness of the high fluorescence intensity region $(\delta)$ in $\mathrm{mm}$. In both cases, $\delta \approx 1.8 \mathrm{~mm}$. The thickness of the high fluorescence intensity region was determined by placing the tissue sample on top of a high resolution scale. Livers of three additional animals served as controls. The first
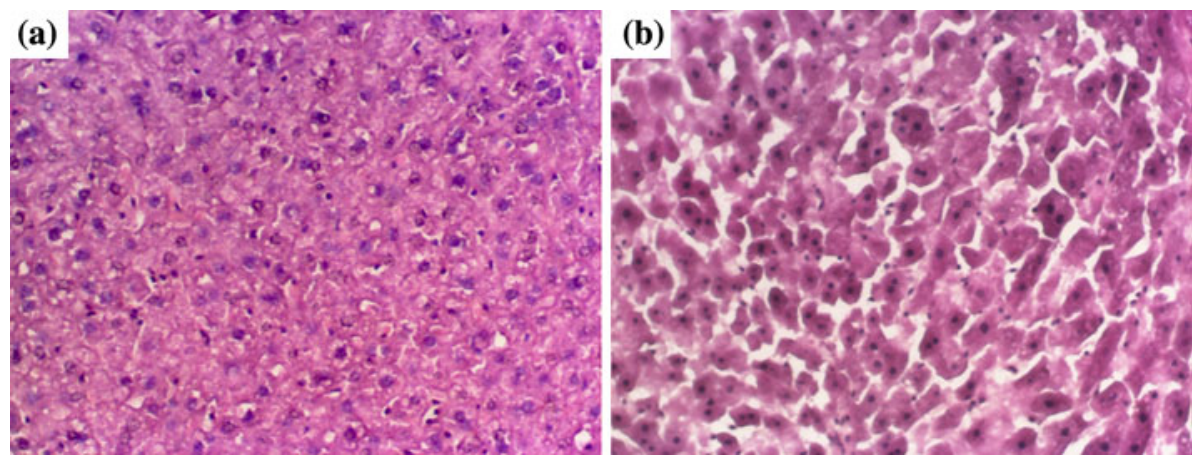

FIGURE 7. (a) Photomicrograph of hematoxylin and eosin stains (200×, H\&E section) showing normal hepatic parenchyma with regular hepatocellular architecture and intact sinusoidal lining. (b) Destruction of vascular structures resulting from cryoinjury indicating hepatic necrosis. 
one was fixed after being excised in $4 \%$ formaldehyde for $5 \mathrm{~h}$ at room temperature, then transferred to a $30 \%$ sucrose solution and incubated at $4{ }^{\circ} \mathrm{C}$ overnight. The second one was imaged immediately after being excised and the third control was stored in DPBS and imaged after $5 \mathrm{~h}$. All the controls showed that all viable cells were found to be positive for GFP expression. In addition, formaldehyde-fixed control tissues showed no degenerative changes and were histologically similar. Figure $8 \mathrm{c}$ shows a control imaged immediately after being excised displaying the same level of fluorescence than the regions of viable tissue in Figs. $8 \mathrm{a}$ and $8 \mathrm{~b}$. Note that in Fig. $8 \mathrm{c}$ the lower section of the control has a slight variation in GFP fluorescence levels. This occurs because when the samples are placed at the microscope stage their height is not even and this results in uneven levels of fluorescence intensity. This also can be seen in Figs. $8 \mathrm{a}$ and $8 \mathrm{~b}$ where the brightness of the upper necrotic tissue is also dimmer. Nevertheless, these slight variations in fluorescence intensity are not significant when observing a clear demarcation between necrotic and viable tissue. Figure $8 \mathrm{~d}$ shows an inverse control imaged after $5 \mathrm{~h}$ of incubation in DPBS after running a LAC procedure without laser heating. The image illustrates the loss of GFP fluorescence in non-viable cells. In order to assess that the incubation in DPBS does not affect fluorescence intensity, after identical LAC procedures, the samples are imaged after 1, 2, 3, and $4 \mathrm{~h}$. A comparison between fluorescent intensities of the imaged samples (not shown) did not show any reduction in GFP fluorescence due to incubation alone. Figure 9 shows the outcome of viability for the same tissue sample after a LAC procedure for different levels of the exposure values (EV) in milliseconds. Detection of low expression levels of the GFP signal was quantified by converting the acquired images to grayscale (16 bits) and assigning a threshold of pixel intensity that corresponds to at least a $50 \%$ reduction of fluorescence relative to the higher peak. Again, although
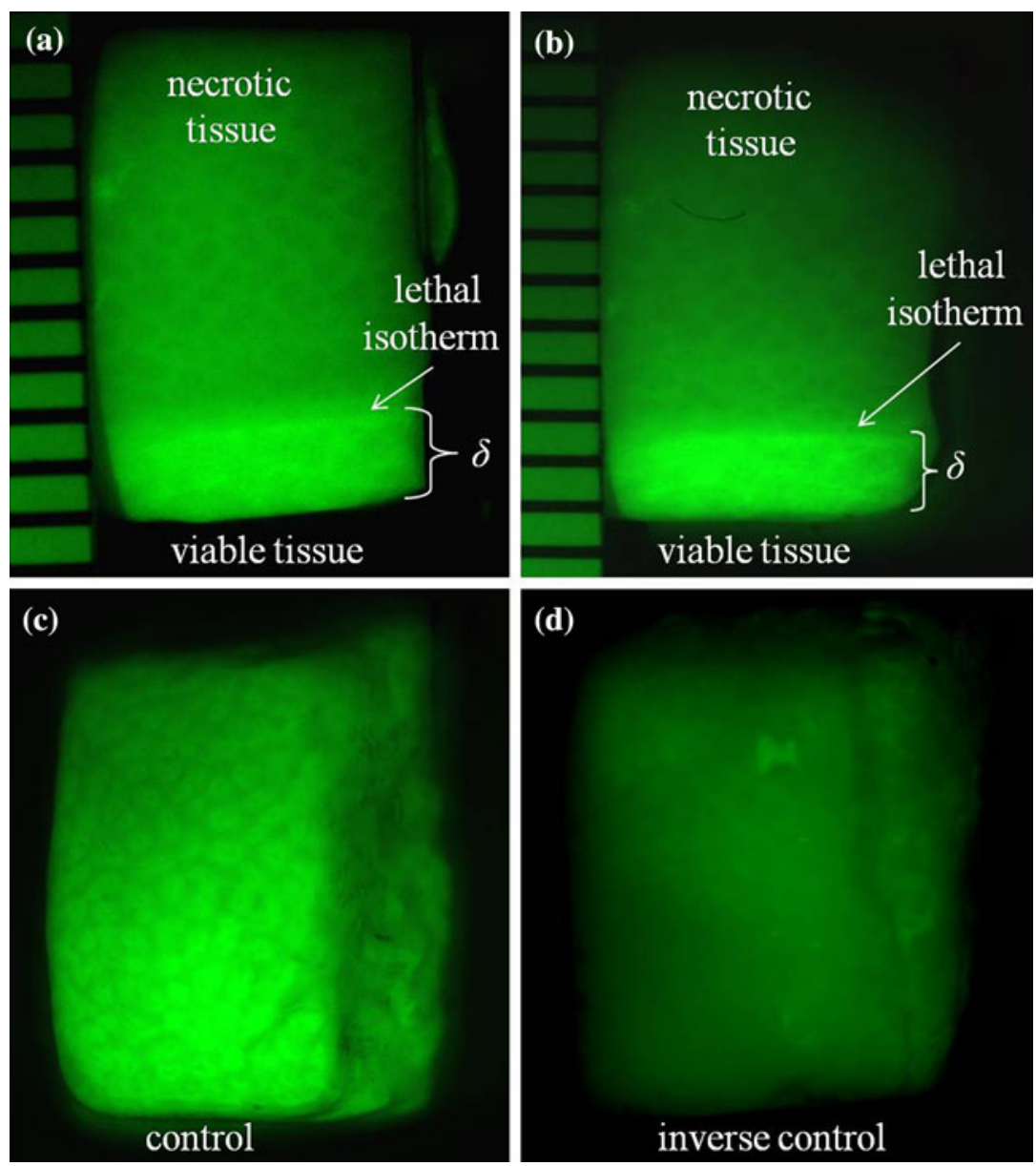

FIGURE 8. High-low intensity contrast in fluorescence. The sharp contrasts in GFP intensity are directly correlated to cell viability in that region. (a, b) GFP viability assays showing a demarcated frontier between viable and necrotic tissue in two different samples assayed after identical LAC procedures. In both cases the thickness of the protected region achieved is the same, and its estimated value is $\delta \approx 1.8 \mathrm{~mm}$. (c) Control sample imaged immediately after excision. (d) Inverse control imaged after $5 \mathrm{~h}$ of incubation in DPBS after running a LAC procedure without laser heating. 

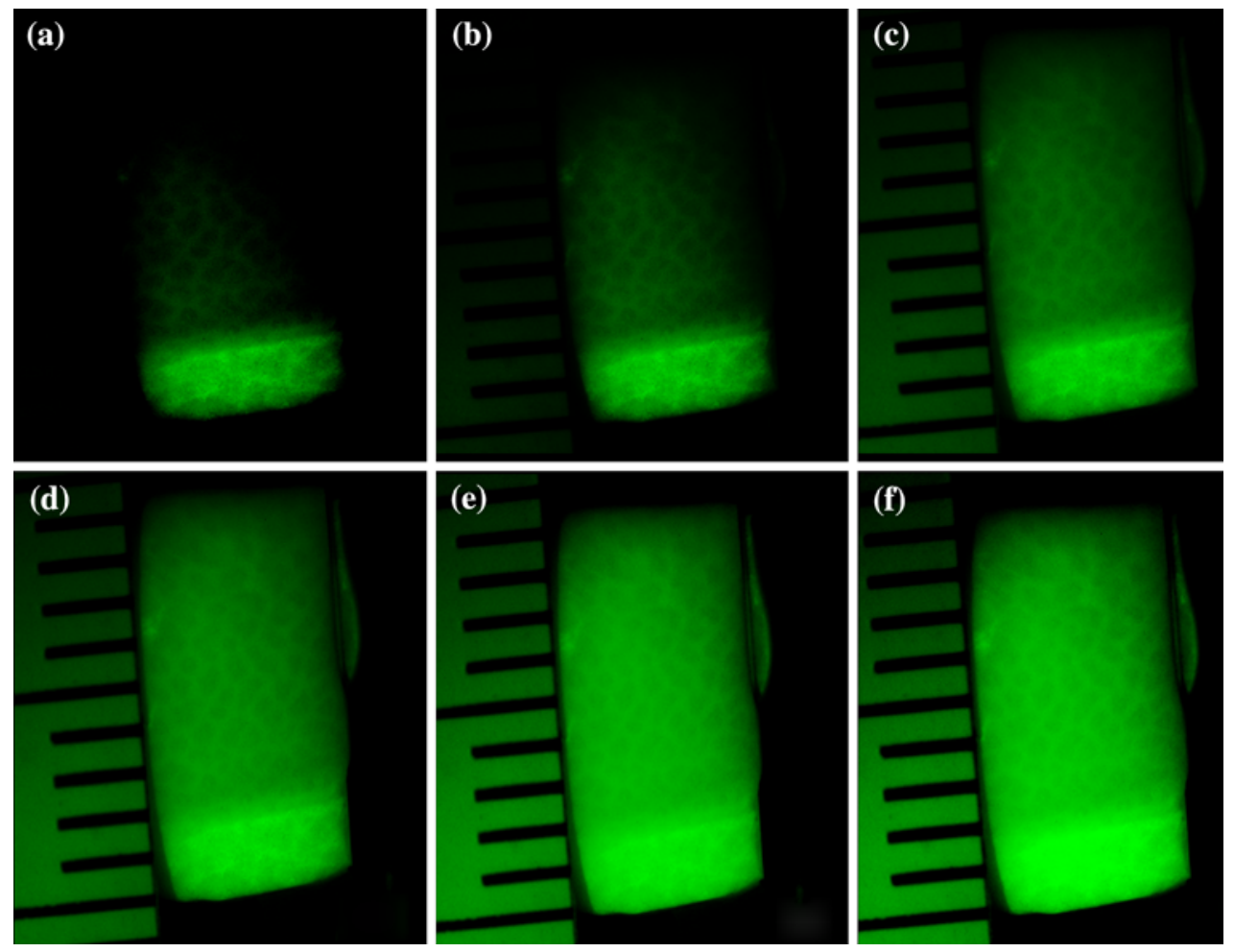

FIGURE 9. Images taken after a LAC procedure for different levels of the exposure values (EV): (a) EV = 50, (b) EV = 100, (c) $E V=150$, (d) $E V=200$, (e) $E V=250$, and (f) $E V=300$.

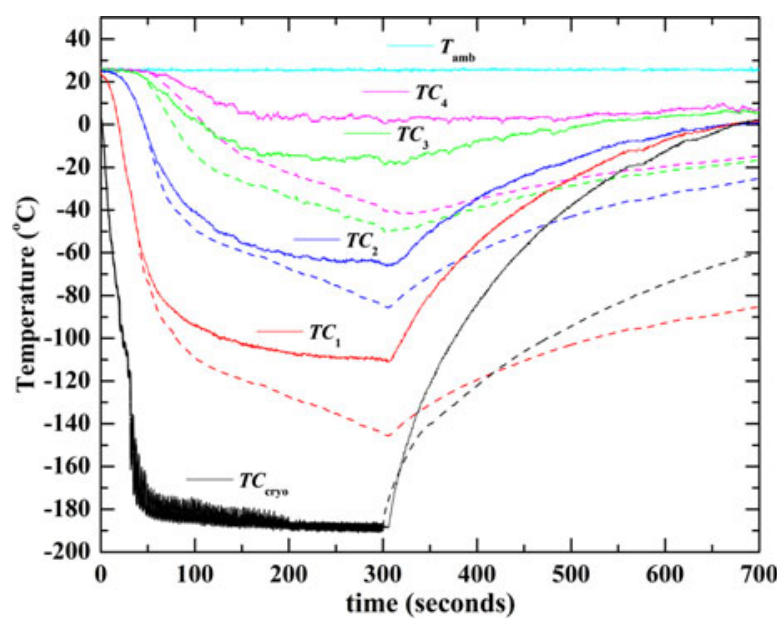

FIGURE 10. Comparison of temperature histories between a LAC procedure (solid lines) and a LAC procedure without laser heating (dashed lines).

the levels of fluorescence intensity throughout the sample show a slight variation, a clear demarcation between necrotic and viable tissue is evident for all the EVs. Figure 10 shows a comparison between the temperature history of a LAC procedure (solid lines) and a LAC procedure without laser heating (dashed lines). The image clearly shows that steeper gradients can be achieved using LAC.

\section{DISCUSSION}

The success of a cryosurgical procedure today is very user-dependent. One of the most substantial challenges in cryosurgery is the incomplete tumor ablation near the ice ball edge, where tissues are frozen but not completely destroyed and, thus, are prone to tumor recurrence. ${ }^{16}$ Since tumor recurrence after surgery should be precluded at all costs, a safety margin of $1 \mathrm{~cm}$ is used to freeze all regions of a small tumor to lethal temperatures. In prostate cryosurgery, it is desirable to set a temperature goal of $-50{ }^{\circ} \mathrm{C}$ in all cancer areas and $-40{ }^{\circ} \mathrm{C}$ at a distance of $1 \mathrm{~cm}$ from the tumor. ${ }^{13,14}$ However, with traditional cryosurgery procedures, this recommendation is difficult to follow in some anatomic locations, such as the prostate gland with its proximity to the rectum and urethra. Results of this study indicate that the above potential problems can be eradicated by LAC implementation. With this new methodology, the freezing injury is significantly enhanced by creating very steep temperature gradients close to the edge of the interface between frozen and unfrozen tissue. Therefore, a lower temperature inside the tumor can be achieved by freezing to temperatures below the lethal threshold with a better enclosure of the tumor within a critical isotherm. In addition, as the distance between the cryoprobe and the optical fiber is 
reduced, steeper gradients can be achieved, since the best protection occurs when the cryoprobe is placed near the protected surface. ${ }^{31}$ Nevertheless, future work in the development of LAC must address the obvious problem of locating the optical fibers with enough precision to achieve the desired gradients. This is an optimization problem, in which the placement of the optical fibers within the tissue and its location relative to the cryoprobes are parameters that should be varied to maximize the superficial tissue protection.

Since the absorption of laser energy is wavelength dependent, the light distribution, penetration depth, and resulting heat generation will depend on the laser of choice. Although this study uses a wavelength of $1320 \mathrm{~nm}$ with an absorption coefficient of $<0.13$ $\left(\mathrm{mm}^{-1}\right),{ }^{24}$ previous studies ${ }^{31,32}$ show that for infrared wavelengths, even when most of the laser energy is absorbed near the surface, the protection achieved by laser heating is still better than the protection achieved by conventional constant temperature heating techniques. In addition, by having proper estimates on the spectral optical properties of prostate, one may better select a laser wavelength that provides a higher penetration depth to increase the thickness of the protected tissue, if required.

Correlation between the temperature histories and the outcome of viability assays presented herein show that the threshold for tissue thermal damage due to cryoinjury using LAC is of $T_{\mathrm{th}} \approx 4{ }^{\circ} \mathrm{C}$ located at a distance of $8.2 \mathrm{~mm}$ away from the cryoprobe tip. Interestingly, this isotherm is located very close to the interface between frozen and unfrozen tissue. This is probably explained because the non-frozen tissue is subject to continued metabolic derangement and the periphery of the frozen tissue has a margin of partially damaged tissue. Therefore, although this region has been exposed to a sublethal freezing insult, this seems to be enough to induce apoptosis. Since the safety distance in cryosurgical procedures may be dramatically reduced with LAC, the requirement to freeze large zones surrounding the tumor to ensure complete destruction may be reduced.

The implementation of LAC may eventually lead to clinical improvements in the outcome of cryosurgery. The implications of its implementation are:

i. Only one freeze/thaw cycle will be necessary. The reason is because once steady-state heat transfer is reached, the entire tumor is encompassed in a lethal isotherm that ensures tissue necrosis.

ii. There will be no need to stop the freezing process prior to achieving the target lethal temperature. iii. The effective cryosurgical tissue destruction zone will be enlarged by producing large thermal gradients, particularly for larger lesions that require overlapping cryoablation zones.

Current research in cryosurgery is concerned with finding a thermal procedure that will absolutely destroy tissue. In this scenario, a new cryosurgical technique has been developed, whereby laser irradiation combines with cryogenic freezing to selectively freeze and better confine damage to cancerous tissue. This is in contrast with most laser tissue heating applications, where lasers are used for ablation and coagulation of soft tissue rather than its preservation. In this study, the thermal history obtained during freezing and thawing in a LAC procedure has been measured. Our experimental results demonstrate the feasibility of LAC for providing thicker and controllable penetration depths. Nevertheless, there are still many issues to address in future research and development of LAC:

i. The time spent at the end-temperature during steady-state heat transfer was not explicitly studied in this work, since we limited this time to $100 \mathrm{~s}$. Further studies are required to verify the influence of the duration of steady-state in the thickness of the protection achieved.

ii. Although LAC is a promising new methodology to treat prostate cancer, significant additional work will be necessary to develop laser power regulation algorithms to control the laser irradiation emitted from the urethra, across its wall and into the prostate. Optimization of laser delivery can be achieved by using a laser system that includes a thermal feedback feature to automatically adjust the laser energy so that a maximum temperature within the tissue never exceeds a specified temperature. In order to obtain improved control, a temperature sensor has been used by some to provide feedback for regulation of the delivered energy, accomplished by switching the laser on or off. ${ }^{10,15,23}$ The sensor can be positioned at the border of the tumor and the temperature kept constant at that position through computer controlled feedback regulation of the laser output.

iii. Development of an accurate deliverance of laser energy needed to achieve the desired protection of the inner wall of the prostate surrounding the urethra. This can be accomplished by using FDA cleared modified fiber optic probes for urethral soft tissue treatment with $360^{\circ}$ or side firing diffusion tips. 
iv. Optimization of the location of optical fibers to enhance the sharpness in the ablative edges must be developed before LAC can be implemented in pre-clinical and clinical trials. This can be accomplished by developing numerical optimization techniques as in previous works. ${ }^{1,2,19,36,37}$

The overall goals, not yet achievable with the results from the present study are to provide a new tool to develop a treatment modality that overcomes the limitations of traditional prostate cancer cryotherapy, especially for large or irregularly shaped tumors. By having the capability to shape the frozen region to strict boundaries within which tissue necrosis is ensured and beyond which viable and healthy tissue prevails, it may be possible to eradicate most of the major complications of cryosurgery, such as sloughing (necrosis) of the prostatic urethra, bladder neck contracture, incontinence, and impotence. As most advances in medical technology, LAC is expected to positively impact the therapeutic outcome and, thus, the quality of life of many patients.

The present results suggest that further studies examining the feasibility of such a procedure in vivo should be pursued. Such studies are currently under way in our laboratory.

\section{ACKNOWLEDGMENTS}

G. Aguilar and L. Martínez-Suástegui would like to acknowledge the support of the UC MEXUSCONACYT program for a Postdoctoral Fellowship granted to the latter to spend a year as a postdoctoral researcher at UCR. The authors thank David R. Hennings CoolTouch CEO for providing the laser system and optical fibers. B. Duperray acknowledges the school of ENSICaen and F. Godinez acknowledges the UCR Undergraduate Research Grant, the UC-LEADS, and the CAMP programs for the support provided to him. G. Guillén acknowledges support of the DGAPA program (UNAM) for a Fellowship to spend an internship at UCR. The authors thank Feng Sun and Florian Langer for their help and efforts with $H \& E$ staining. In particular, the authors appreciate the input of A.M. Walker from the University of California Riverside, Division of Biomedical Sciences and S. Robert from the VIB Department of Plant Systems Biology, Ghent University. The authors gratefully acknowledge expert animal husbandry by Ms. Sally Scott. This work was supported in part by NSF under grant NSF-CTS-SGER: 0609662.

\section{OPEN ACCESS}

This article is distributed under the terms of the Creative Commons Attribution Noncommercial License which permits any noncommercial use, distribution, and reproduction in any medium, provided the original author(s) and source are credited.

\section{REFERENCES}

${ }^{1}$ Baissalov, R., G. A. Sandison, B. J. Donnelly, J. C. Saliken, J. G. McKinnon, K. Muldrew, and J. C. Rewcastle. A semi-empirical treatment planning model for optimization of mutiprobe cryosurgery. Phys. Med. Biol. 45:10851098, 2000.

${ }^{2}$ Baissalov, R., G. A. Sandison, D. Reynolds, and D. Muldrew. Simultaneous optimization of cryoprobe placement and thermal protocol for cryosurgery. Phys. Med. Biol. 46:1799-1814, 2001.

${ }^{3}$ Baumstark-Khan, C., M. Palm, J. Wehner, M. Okabe, M. Ikawa, and G. Horneck. Green fluorescent protein (GFP) as a marker for cell viability after UV irradiation. J. Fluoresc. 9:37-43, 1999.

${ }^{4}$ Bischof, J. C., N. Merry, and J. Hulbert. Rectal protection during prostate cryosurgery: design and characterization of an insulating probe. Cryobiology 34:80-92, 1997.

${ }^{5}$ Chalfie, M., Y. Tu, G. Euskirchen, W. W. Ward, and D. C. Prasher. Green fluorescent protein as a marker for gene expression. Science 263:802-805, 1994.

${ }^{6}$ Chang, Z., J. J. Finkelstein, H. Ma, and J. G. Baust. Development of high-performance multiprobe cryosurgical device. Biomed. Instrum. Technol. 28:383-390, 1994.

${ }^{7}$ Cody, C. W., D. C. Prasher, W. M. Westler, F. G. Prendergast, and W. W. Ward. Chemical structure of the hexapeptide chromophore of the Aequorea green fluorescent protein. Biochemistry 32:1212-1218, 1993.

${ }^{8}$ Cohen, T. K., R. J. Miller, and B. A. Shumarz. Urethral warming catheter for use during cryoablation of the prostate. Urology 45:861-864, 1995.

${ }^{9}$ Collins, L. A., M. N. Torrero, and S. G. Franzblau. Green fluorescent protein reporter microplate assay for high-throughput screening of compounds against Mycobacterium tuberculosis. Antimicrob. Agents Chemother. 42: 344-347, 1998

${ }^{10}$ Daikuzono, N., S. Suzuki, H. Tajiri, H. Tsunekawa, M. Ohyama, and S. N. Joffe. Laserthermia: a new computer-controlled contact Nd:YAG system for interstitial local hyperthermia. Lasers Surg. Med. 8:254-258, 1988.

${ }^{11}$ Elliot, G., J. McGrath, and E. Crockett-Torabi. Green fluorescent protein: a novel viability assay for cryobiological applications. Cryobiology 40:360-369, 2000.

${ }^{12}$ Gage, A. A. Critical temperature for skin necrosis in experimental cryosurgery. Cryobiology 19:273-281, 1982.

${ }^{13}$ Gage, A. A., and J. G. Baust. Mechanisms of tissue injury in cryosurgery. Cryobiology 37:171-186, 1998.

${ }^{14}$ Hoffmann, N. E., and J. C. Bischof. The cryobiology of cryosurgical injury. Urology Suppl 2A:40-49, 2002.

${ }^{15}$ Ivarsson, K., J. Olsrud, C. Sturesson, P. H. Möller, B. R. Persson, and K.-G. Tranberg. Feedback interstitial diode laser $(805 \mathrm{~nm})$ thermotherapy system: ex vivo evaluation and mathematical modeling with one and fourfibers. Lasers Surg. Med. 22:86-96, 1998. 
${ }^{16}$ Larson, T. R., D. W. Robertson, A. Corica, and D. G. Bostwick. In vivo interstitial temperature mapping of the human prostate during cryosurgery with correlation to histopathologic outcomes. Adult Urol. 55:547-552, 2000.

${ }^{17}$ Leff, L. G., and A. A. Leff. Use of green fluorescent protein to monitor survival of genetically engineered bacteria in aquatic environments. Appl. Environ. Microbiol. 62:34863488, 1996.

${ }^{18}$ Lehtinen, J., J. Nuutila, and E. Lilius. Green fluorescent protein-propidium iodide (GFP-PI) based assay for flow cytometric measurement of bacterial viability. Cytometry $A$ 60:165-172, 2004.

${ }^{19}$ Lung, D. C., T. F. Stahovich, and Y. Rabin. Computerized planning for multiprobe cryosurgery using a force-field analogy. Comput. Methods Programs Biomed. 7:101-110, 2004.

${ }^{20}$ Mala, T., L. Aurdal, L. Frich, E. Samset, P. K. Hol, B. Edwin, O. Soreide, and I. Gladhaug. Liver tumor cryoalbation: a commentary on the need of improved procedural monitoring. Technol. Cancer Res. Treat. 3:85-91, 2004.

${ }^{21}$ Masters, A., and S. G. Brown. Interstitial laser hyperthermia in tumour therapy. Ann. Chir. Gynaecol. 79:244-251, 1990.

${ }^{22}$ Masters, A., and S. G. Brown. Interstitial laser hyperthermia. Semin. Surg. Oncol. 8:242-249, 1992.

${ }^{23}$ Möller, P. H., L. Lindberg, P. H. Hendriksson, B. R. R. Persson, and K.-G. Tranberg. Temperature control and light penetration in a feedback laser thermotherapy system. Int. J. Hyperth. 12:49-63, 1996.

${ }^{24}$ Müller, G. J., and A. Roggan. Laser-Induced Interstitial Thermotherapy. Bellingham, WA: SPIE The International Society for Optical Engineering, 1995.

${ }^{25}$ Onik, G. M., J. K. Cohen, G. D. Reyes, B. Rubinsky, Z. H. Chang, and J. G. Baust. Transrectal ultrasoundguided percutaneous radical cryosurgical ablation of the prostate. Cancer 72:1291-1299, 1993.

${ }^{26}$ Prasher, D. C., V. K. Eckenrode, W. W. Warde, F. G. Prendergast, and M. J. Cormier. Primary structure of the Aequorea victoria green-fluorescent protein. Gene 11:229-233, 1992.
${ }^{27}$ Rabin, Y., and A. Shitzer. A new cryosurgical device for controlled freezing, part I: setup validation test. Cryobiology 33:82-92, 1996.

${ }^{28}$ Rabin, Y., and T. F. Stahovich. The thermal effect of urethral warming during cryosurgery. Cryoletters 23:361-374, 2002.

${ }^{29}$ Rabin, Y., and T. F. Stahovich. Cryoheater as a means of cryosurgery control. Phys. Med. Biol. 48:619-632, 2003.

${ }^{30}$ Rabin, Y., R. Coleman, D. Mordohovich, R. Ber, and A. Shitzer. A new cryosurgical device for controlled freezing, part II: in vivo experiments on rabbits' hind thighs. Cryobiology 33:93-105, 1996.

${ }^{31}$ Romero-Méndez, R., K. Chu, H. Vu, W. Franco, and G. Aguilar. Confinement of freezing front by laser irradiation during cryosurgery. In: Proceedings of 2004 ASMESummer Heat Transfer Conference, San Francisco, CA, 2005.

${ }^{32}$ Romero-Méndez, R., W. Franco, and G. Aguilar. Laserassisted cryosurgery of prostate: numerical study. Phys. Med. Biol. 52:463-478, 2007.

${ }^{33}$ Shroder, T., M. Castren-Persons, A. Lehtinen, and M. Taavitsainen. Percutaneous interstitial laser hyperthermia in clinical use. Ann. Chir. Gynaecol. 83:286-290, 1994.

${ }^{34}$ Steger, A. C., W. R. Lees, K. Walmsley, and S. G. Brown. Interstitial laser hyperthermia: a new approach to local destruction of tumours. Br. Med. J. 299:362-365, 1989.

${ }^{35}$ Strebel, A., T. Harr, F. Bachmann, M. Wernli, and P. Erb. Green fluorescent protein as a novel tool to measure apoptosis and necrosis. Cytometry 43:126-133, 2001.

${ }^{36}$ Tanaka, D., K. Shimada, M. R. Rossi, and Y. Rabin. Cryosurgery planning using bubble packing in 3D. Comput. Methods Biomech. Biomed. Eng. 11:113-121, 2007.

${ }^{37}$ Tanaka, D., K. Shimada, M. R. Rossi, and Y. Rabin. Towards intra-operative computerized planning of prostate cryosurgery. Int. J. Med. Robot. Comput. Assist. Surg. 3:10-19, 2007.

${ }^{38}$ Unge, A., R. Tombolini, L. Molbak, and J. K. Jansson. Simultaneous monitoring of cell number and metabolic activity of specific bacterial populations with a dual gfp-luxAB marker system. Appl. Environ. Microbiol. 65: 813-821, 1999. 